Revue d'histoire de l'Amérique française

AH REVUE D.HISTOIRE DE L'AMÉRIQUE FRANÇAISE

\title{
Les évêques canadiens contre le projet d'Union des deux Canadas (1822-1824)
}

\section{Lucien Lemieux}

Volume 22, numéro 3, décembre 1968

URI : https://id.erudit.org/iderudit/302797ar

DOI : https://doi.org/10.7202/302797ar

Aller au sommaire du numéro

Éditeur(s)

Institut d'histoire de l'Amérique française

ISSN

0035-2357 (imprimé)

1492-1383 (numérique)

Découvrir la revue

Citer cet article

Lemieux, L. (1968). Les évêques canadiens contre le projet d'Union des deux Canadas (1822-1824). Revue d'histoire de l'Amérique française, 22(3), 393-400. https://doi.org/10.7202/302797ar d'utilisation que vous pouvez consulter en ligne.

https://apropos.erudit.org/fr/usagers/politique-dutilisation/ 


\section{LES EVEQUES CANADIENS CONTRE LE PROJET D'UNION DES DEUX CANADAS}

(1822-1824)

Le problème le plus important auquel le gouverneur Dalhousie eut à faire face durant son séjour à Québec fut celui de l'Union des deux Canadas. Le partage des recettes douanières entre ces deux colonies se faisait depuis longtemps au détriment du Bas-Canada; son administration en souffrait. ${ }^{1}$ De plus, les relations entre le gouverneur et la Chambre d'Assemblée étaient souvent tendues, surtout à cause de la question des subsides. Mgr Plessis, alors évêque de Québec, n'aimait pas ce genre de querelles parlementaires: elles finiraient peut-être au préjudice des Canadiens dont les vues étaient bonnes, mais qui ne prenaient pas suffisamment en considération les circonstances dans lesquelles ils se trouvaient '2. Quand l'idée de l'Union fit son apparition, le gouverneur y donna tout son appui. En juin, à Londres, Ellice réussit à faire porter le projet aux Communes. Cet item était inclus dans un ensemble de problèmes à résoudre, tels que le commerce à régulariser et les gouvernements des deux Canadas à aider plus efficacement. James MacIntosh, dans un plaidoyer habile et résolu, fit observer que le Parlement se devait de consulter au préalable les sentiments du peuple canadien que l'on avait laissé dans l'ignorance des débats en cours, spécialement pour ce qui concernait l'Union. Les articles relatifs à cette

${ }^{1}$ D. G. Creighton dans The Commercial Empire of the St. Lawrence, 1760-1850 (Toronto, The Ryerson Press, 1937), 105, divise l'histoire des conditions commerciales de l'Amérique Britannique du Nord entre 17601850 en trois périodes: $1760-1783,1783-1821$, période de transition, et 1821-1850. Il caractérise cette dernière par l'abolition du vieux système colonial et l'installation du gouvernement responsable. Trois aspects sont envisagés: 1) la compétition croissante des Etats-Unis; 2) la disparition lente du vieux système colonial; 3) l'intensification de l'inadaptation économique et de conflits sociaux.

2 AHWL (Archbishop's House, Westminster, London), Quebec (Montreal), 24 janv. 1822, Plessis à Poynter. 
question furent donc soustraits, et les parlementaires s'en tinrent à The Canada Trade Act. Un projet de loi distinct, concernant l'Union, devait être présenté à la session suivante. Un bill fut préparé dont les points principaux consistaient en ceci : réunion des membres des deux Conseils législatifs en un seul et des membres des deux Chambres d'Assemblée en une seule; tous les écrits de ce Conseil unique et de cette Chambre unique seraient faits en langue anglaise comme tous les débats devraient l'être aussi quinze ans après la passation de l'Acte; et la nomination aux cures des paroisses catholiques romaines sera ni plus ni moins soumise au gouverneur.

Quand, en septembre, la nouvelle de cette tentative d'Union parvint au Canada, les réactions surgirent de partout. Grâce aux deux registres de Jacques Viger, secrétaire du comité de la ville contre le bill d'Union, on connaît les démarches entreprises dans les deux Canadas pour préparer une pétition contre le projet. Dès le 7 octobre, au Champ de Mars, à Montréal, une première réunion publique permit aux intéressés de prendre sept résolutions qui servirent de modèles par la suite à toutes celles qui furent prises dans les assemblées des divers comtés et paroisses. Louis-Joseph Papineau fut nommé président du comité de Montréal ${ }^{3}$. Des projets de pétition furent proposés. A Québec, on fusionna en un seul les quatre principaux projets, et l'on fit de même ailleurs par la suite. Il s'agissait de faire signer cette pétition par le plus grand nombre de personnes possible. D'autres groupes nuancèrent leur opposition ou leur adhésion au bill. Pour leur part, les partisans de l'Union se recrutèrent parmi les Britanniques des deux Canadas. La réalisation $d u$ projet leur paraissait indispensable aux points de

${ }^{3}$ L.-P. Desrosiers, "Montréal soulève la Province", dans Les Cahiers des Dix, 8 (1943): 75. Ces deux registres étaient intitulés: Procédés des habitants de la Cité et du District de Montréal, amis de la Constitution des Canadas de 1791, aux fins de concerter des moyens de la conserver intacte et de prévenir, si possible, la passation du "Bill d'Union des Législatures du Haut et du Bas-Canada, tel que projeté et amendé par un Comité de la Chambre des Communes, le 31 juillet 1822"; contenant la correspondance du Comité Général de ce District avec ceux des districts de Québec et des Trois-Rivières, et plusieurs des Comités Constitutionnels du Haut-Canada. Avec Appendices. 
vue politique, économique et démographique; en fait, ils avaient surtout en vue d'en arriver à une population majoritaire de langue anglaise. Les British Montrealers prônèrent la méthode forte, afin de n'être entravés d'aucune façon par les Canadiens; les Britanniques des Cantons de l'est et ceux de Québec désiraient plutôt la subordination législative des Canadiens dont la présence était cependant requise comme un peuple-rempart vis-à-vis des Etats-Unis. Il se trouva aussi parmi les Britanniques un petit nombre de modérés (James Stuart en fit partie) et même des adversaires de l'Union. Ces derniers étaient soit des fonctionnaires craignant de perdre leur toute-puissance ou leurs privilèges, soit des citoyens du Haut-Canada ayant peur d'être soumis aux Canadiens français qui seraient en majorité dans l'ensemble des provinces, soit d'autres individus qui ne croyaient pas l'Union nécessaire pour régler le partage des douanes et la question des subsides. Quant aux Canadiens, ils s'opposèrent en bloc à l'Union, parce qu'elle était voulue par une minorité qui n'admettait pas leur influence dans l'orientation politique du BasCanada, et parce qu'elle était une menace d'anglicisation ${ }^{4}$.

Mgr Plessis ne pouvait rester indifférent devant ces remous qui balayaient les deux provinces. Son diocèse comprenait encore tout ce territoire, bien qu'il fût aidé dans la région de Montréal par Mgr Lartigue, et dans le Haut-Canada par Mgr Macdonell, tous deux vicaires généraux, évêques auxiliaires et suffragants de l'évêque de Québec. Très heureux que le projet d'Union n'eût pas passé aux Communes en juin et en juillet, Mgr Plessis invita Mgr Poynter à surveiller la marche de l'affaire à Londres, spécialement à propos de l'article concernant l'Église catholique, qui stipulait que nul ecclésiastique catholique (il s'agissait surtout des curés) n'aurait droit désormais à ses revenus, à moins d'être muni d'une commission royale et de consentir à diverses autres exigences. Il s'attendait à ce que le vicaire apostolique de Londres se trouvât assez d'amis pour empêcher la ratification d'un tel article, lequel apparaîtrait d'ailleurs comme un relent de

${ }^{4}$ D. Vaugeois, L'Union des deux Canadas. Nouvelle Conquête? (17911840) (Trois-Rivières, Edit. du Soc, 1962), 63-91; il s'agit du chapitre IV, intitulé: "Tentative d'Union: 1822". 
la mentalité de 1700 contre la religion catholique ${ }^{5}$. L'évêque de Québec ne se limita pas à cette activité. Il signa à la fin de novembre les adresses envoyées au roi et aux deux Chambres du Parlement impérial contre le bill d'Union, telles que rédigées d'un commun accord par les comités de Montréal et de Québec. Les membres du clergé de Québec en firent autant. Mgr Plessis invita Mgr Lartigue à suivre son exemple ${ }^{6}$. Ce dernier acquiesça. Il aurait même désiré que Mgr Plessis allât plus loin; après avoir lu des pétitions d'unionistes, des commentaires dans les journaux et la fameuse clause 25e du bill, laquelle soumettait la nomination des curés à l'approbation du gouverneur, Mgr Lartigue engagea l'évêque de Québec à s'expliquer une fois pour toutes avec les représentants du gouvernement britannique sur la prétendue suprématie du roi sur l'Église catholique du Canada. Bien que l'on s'en référât toujours à l'Acte de Québec à ce propos, il ne lui semblait pas difficile de prouver que cette clause était nulle de plein droit, parce que contraire à une des conditions de la capitulation du pays en 1763 . Celle-ci avait assuré aux nouveaux sujets britanniques la liberté de leur religion, laquelle liberté était incompatible avec la suprématie royale ${ }^{7}$. Mgr Plessis fit remarquer à son auxiliaire de Montréal que Mgr Denaut, son prédécesseur sur le siège de Québec, avait dû chaque année rendre compte au gouverneur des changements survenus dans les cures.

Tout cet assujettissement me déplaisait fort. Il fallut néanmoins m'y soumettre dans les premières années de mon épiscopat, et ce n'est que sous l'administration de Sir George Prevost que j'ai pleinement réussi à m'en affranchir, après l'avoir inutilement tenté avec $M$. Dunn et le général Craig. Ce sont là des choses qu'il est bon que vous sachiez, mais dont il est à propos que le clergé ne soit pas imbu.

Mgr de Québec tenait à éviter tout ce qui pourrait entraîner des répercussions malheureuses et imprudentes contre l'autorité

5 AHWL, Quebec (Montreal), 16 nov. 1822, Plessis à Poynter.

6 ACAM (Archives de la Chancellerie de l'Archevêché de Montréal), 295.101, 483-486, 25 nov. 1822, Plessis à Lartigue.

7 AAQ (Archives de l'Archevêché de Québec), Evêché de Montréal, I: 160-161, 30 déc. 1822, Lartigue à Plessis. 
civile; il valait mieux attendre avant de donner suite aux propositions de Mgr Lartigue ${ }^{8}$.

Deux agents, l'un de Montréal et l'autre de Québec, furent nommés pour porter à Londres la requête des Canadiens des deux Canadas: Louis-Joseph Papineau et John Neilson. Déjà le 17 décembre 1822, Dalhousie avait envoyé à Bathurst, secrétaire d'État aux Colonies, une copie de la pétition opposée à l'Union alors en circulation dans les deux provinces ${ }^{9}$. Le 6 janvier, Papineau avertit Cochran, secrétaire de Dalhousie, qu'il ne pourrait pas se rendre à l'ouverture du parlement provincial quatre jours plus tard, ni remplir sa charge d'Orateur de la Chambre d'Assemblée durant la session à venir. On l'avait sollicité pour accomplir une tâche très délicate et de grande portée: le maintien de la Constitution, qu'à si juste titre les habitants de la colonie regardaient comme la sauvegarde de tous leurs droits ${ }^{10}$. Mgr Lartigue écrivit au vicaire apostolique de Londres pour lui présenter les deux agents des Canadiens, tous deux membres du parlement provincial. A cause de la clause concernant l'Église catholique, il incita lui aussi Mgr Poynter à user de son influence afin que le bill ne passât pas. Il lui proposa d'introduire Papineau et Neilson à lord Clifford qui les présenterait peut-être au duc de Sussex. Ne pourraient-ils pas rencontrer de plus Charles Butler, le savant homme de loi anglais ${ }^{11}$ ? Ils quittèrent Montréal le 17 janvier en direction de l'Angleterre, par la voie de New-York. Les diverses pétitions anti-unionistes dont ils étaient chargés avaient été signées par 69,404 personnes, dont 8,762 du Haut-Canada. Un retard d'organisation dans le district des Trois-Rivières y limita le nombre des signataires à 6,000. Beaucoup de ces signatures étaient des $X$ joints aux noms des individus concernés.

Pour sa part, Dalhousie envoya lui-même à Bathurst la requête des habitants du district de Montréal favorables à l'Union.

8 ACAM, 295.101, 512-519, 27 janv. 1823, Plessis à Lartigue.

9 PROL (Public Record Office, London), CO 42, 191-II, 294-299.

10 PROL, CO 42, 196, 20 (copie).

11 AHWL, Quebec (Montreal), 12 janv. 1823. 
Par une autre voie plus sûre et plus rapide, il fit parvenir au roi l'original de cette requête et des signatures. Les signataires étaient tous des Britanniques. En même temps, il expédia l'adresse du Conseil législatif bas-canadien. Fait surprenant, les membres de ce Conseil, sans avoir été influencés par l'attitude anti-unioniste de l'Assemblée, avaient voté contre l'Union au nombre de quatorze contre six. D'après eux l'Union aurait été la source inévitable de maux plus grands et aurait tendu à affaiblir l'administration du gouvernement de Sa Majesté ${ }^{12}$. Les unionistes déléguèrent tout de même un représentant à Londres dans la personne du modéré James Stuart.

Arrivés en février dans la capitale britannique, les délégués des deux groupes eurent le temps de se concerter. Rien ne se fit au parlement, et Adam Lymburner, un ami de Mgr Plessis très au courant des affaires parlementaires, prévoyait que le bill ne serait pas débattu durant l'année en cours. En effet, l'opposition entre les différents goupes au Canada intéressait très peu les politiciens britanniques. Ils étaient encore sur le qui-vive à propos de la participation de la Grande-Bretagne à la guerre qui menaçait de se déclarer entre la France et l'Espagne. Lymburner assura du moins l'évêque de Québec que rien ne serait voté au détriment de l'Église catholique ni de ses adhérents ${ }^{13}$. Le 12 mai, Papineau et Neilson remirent à Horton, sous-secrétaire d'État aux Colonies, toutes les remarques et observations contraires au bill projeté. Il fut entendu que la requête des habitants du Bas-Canada contre le bill d'Union serait présentée à la Chambre des lords par Bathurst et à celle des Communes par Horton, et que celle des habitants du Haut-Canada serait présentée par James MacIntosh. Neilson rapporta ces événements et ces décisions au comité de Montréal dès son retour le 25 juin au soir. Quand Papineau eut la certitude que ses documents avaient été soumis au Cabinet par Bathurst lui-même (comme ceux de Stewart d'ailleurs) ${ }^{14}$, qu'il eut assisté aux

12 PROL, $C O$ 42 196, 48-49, 24 janv. 1823 (orig. angl.).

13 AAQ, Angleterre, III: 107, 8 avril 1823, Lymburner à Plessis (orig. angl.). angl.).

14 PROL, CO 43, 63, 79-81, 24 juillet 1823, Horton à Papineau (orig. 
séances du parlement, qu'il eut constaté qu'on n'y avait pas parlé du bill d'Union, et qu'il fut assuré qu'on n'en parlerait pas à l'avenir sans que les Canadiens ne fussent informés d'avance par le gouverneur général des deux Canadas, il revint à Montréal le 25 novembre 1823. On pouvait espérer, disait-il, que le bill ne serait jamais voté au parlement britannique. Le ministre Bathurst l'avait assuré que si jamais il passait, on en retrancherait la clause qui concernait la religion catholique et celle qui prétendait enlever l'usage du français écrit ou parlé en politique ${ }^{15}$.

Vers la même époque, le ministre aux Colonies reçut une lettre de Dalhousie dans laquelle celui-ci revenait sur l'importance de l'Union. Sans aller aussi loin que Stewart sur certains points, il était d'avis, avec lui, qu'on avait pris des moyens et des artifices plus ou moins honnêtes pour recueillir les signatures contre le bill, que les sentiments des Canadiens du Bas-Canada ne concordaient aucunement avec ce que Neilson et Papineau avaient indiqué, que les Canadiens accepteraient avec gratitude la décision du parlement impérial en faveur de l'Union, et que de toute évidence la prospérité de la province en dépendait. En outre,

les prérogatives du roi comme chef de l'Eglise doivent être exercées dans cette province tout autant à l'intérieur de l'Eglise catholique qu'à l'intérieur de l'Eglise protestante, au moins pour ce qui concerne l'établissement des paroisses et la nomination des pasteurs ${ }^{16}$.

Une année d'accalmie s'écoula. Une lettre de Peter Burnett ranima l'opposition des Canadiens au projet de l'Union. Ce Burnett avertit le comité anti-unioniste de Québec que le bill serait présenté à la Chambre des communes à la session suivante. Mgr Plessis, mis au courant, entretint Mgr Poynter là-dessus. Il craignait que l'on introduisît une clause injurieuse à la liberté de l'Église catholique. Une lettre envoyée l'année précédente à Liverpool avait dévoilé le système habituel des adversaires des

15 AHWL, Quebec (Montreal), 15 déc. 1823, Lartigue à Poynter.

16 PROL, CO 42, 196, 296-300, 21 nov. 1823 (orig. angl.). 
deux Canadas contre l'Église catholique, c'est-à-dire celui d'exagérer l'indépendance du clergé, l'autorité de l'évêque et ses revenus. Le clergé protestant admettait difficilement que l'évêque catholique nommât aux cures. Ces adversaires du catholicisme ne savaient-ils pas qu'il en était ainsi dans tous les pays où se trouvaient des catholiques ${ }^{17}$ ? Le 11 novembre 1824 , une réunion spéciale eut lieu à Montréal à la demande du comité de Québec. Comme le gouverneur avait quitté Québec peu auparavant pour se rendre en Angleterre et qu'il ne pouvait ni confirmer ni contredire les prédictions de Burnett, les chefs anti-unionistes crurent en l'avertissement de ce dernier et s'organisèrent à peu près comme deux ans auparavant. De nouveaux documents furent envoyés à MacIntosh, désigné comme leur agent officiel à Londres. Ce dernier leur répondit seulement en septembre de l'année suivante. Il avait été gravement malade; sa convalescence avait été longue et il n'avait pu participer aux réunions du parlement ${ }^{18}$. En réalité, rien de nouveau ne s'était produit à Londres; d'ailleurs, les affaires canadiennes reçurent un minimum d'attention durant les années 1822-1828. Seuls les deux actes: Canada Trade Act de 1822 et Canada Tenures Act de 1825 furent présentés par Horton aux Communes. Ainsi se terminait ce projet d'Union de 1822 . Celui de 1840 aurait plus de succès, malgré l'opposition de l'épiscopat canadien du temps.

Université de Montréal

LUCIEN LEMIEUX

\section{Univorsite do Montreal}

${ }^{17}$ AHWL, Quebec (Montreal), 28 oct. 1824, Plessis à Poynter.

18 Beaucoup de renseignements rapportés ici à propas du bill d'Union, furent tirés de l'article de L.-P. Desrosiers, "Montréal soulève la Province", dans les Cahiers des Dix, 8 (1943) : 75-107. 